\title{
The X-ray cycle in the solar-type star HD 81809
}

\section{XMM-Newton observations and implications for the coronal structure}

\author{
F. Favata ${ }^{1}$, G. Micela ${ }^{2}$, S. Orlando ${ }^{2}$, J. H. M. M. Schmitt ${ }^{3}$, S. Sciortino ${ }^{2}$, and J. Hall ${ }^{4}$ \\ 1 European Space Agency, 8-10 rue Mario Nikis, 75738 Paris Cedex 15, France \\ e-mail: Fabio.Favata@esa.int \\ 2 INAF - Osservatorio Astronomico di Palermo, Piazza del Parlamento 1, 90134 Palermo, Italy \\ 3 Universität Hamburg, Hamburger Sternwarte, Gojenbergsweg 112, 21029 Hamburg, Germany \\ ${ }^{4}$ Lowell Observatory, 1400 West Mars Hill Road, Flagstaff, Arizona, USA
}

Received 3 March 2008 / Accepted 21 April 2008

\section{ABSTRACT}

\begin{abstract}
Context. The 11-yr cycle is the best known manifestation of the Sun's activity. While chromospheric cycles have been studied in a number of solar-like stars, very little is known about how these are reflected in the cyclical behavior of the coronal X-ray emission in stars other than the Sun.

Aims. Our long-term XMM-Newton program of long-term monitoring of a solar-like star with a well-studied chromospheric cycle, HD 81809, aims to study whether an X-ray cycle is present, along with studying its characteristics and its relation to the chromospheric cycle.

Methods. Regular observations of HD 81809 were performed with XMM-Newton, spaced by 6 months from 2001 to 2007 . We studied the variations in the resulting coronal luminosity and temperature, and compared them with the chromospheric Ca II variations. We also modeled the observations in terms of a mixture of active regions, using a methodology originally developed to study the solar corona.

Results. Our observations show a well-defined cycle with an amplitude exceeding 1 dex and an average luminosity approximately one order of magnitude higher than in the Sun. The behavior of the corona of HD 81809 can be modeled well in terms of varying coverage of solar-like active regions, with a larger coverage than for the Sun, showing it to be compatible with a simple extension of the solar case.
\end{abstract}

Key words. X-rays: stars - stars: activity - stars: coronae - stars: late-type

\section{Introduction}

Cyclic modulation in the activity level appears to be relatively common among solar-type stars. The best known and studied example is of course the Sun itself, for which the 11-yr cycle was noticed in the form of periodic modulation of the sunspot number already in the mid 19th century. Most activity indicators follow similar cyclical variations (although the study of the Sun's magnetic field shows the true cycle to be $22 \mathrm{yr}$, with the field polarity reversing each cycle), with the amplitude of the modulation depending strongly on the indicator used. The pioneering and persistent work of $\mathrm{O}$. Wilson allowed the detection of chromospheric activity cycles on stars other than the Sun, as amply discussed by Baliunas et al. (1995).

Chromospheric activity cycles appear to be relatively common in intermediate-activity solar type stars, while less active stars are often found in "Maunder minimum"-like states, i.e. showing very little long-term variability (Baliunas et al. 1995). The most active stars appear dominated by irregular variability rather than by cyclic behavior. It is, however, not clear if the "Maunder minimum like" stars, as well as the Sun itself during the Maunder mininimum, are magnetically less active than the modern solar minimum seems to be, even though they show no HK cycle (Judge \& Saar 2007). The present-day Sun has a typical chromospheric modulation amplitude, along the 11-yr cycle, of about a factor of 2 (as measured in the $S$ index).
Evidence of cyclical variations in the coronal emission of stars other than the Sun has so far been elusive. In the Sun the $\mathrm{X}$-ray luminosity follows a cycle with the same periodicity as the chromospheric cycle, but with a much larger amplitude, up to a factor of 100 in the Yohkoh 0.73-2.5 keV band (Acton 1996). Stars with a high activity level, which have been the typical target of X-ray observations, show modest amounts of long-term variability, with no evidence of a cyclic behavior, as for example reviewed by Stern (1998), and as confirmed from a statistical analysis of the ROSAT All Sky Survey data by Schmitt \& Liefke (2004). Evidence of long-term X-ray variability with amplitude of a factor of 2.5 , and correlated with the chromospheric $S$ index, was reported by Hempelmann et al. (2003) in $61 \mathrm{Cyg}$ A and $\mathrm{B}(\mathrm{K} 5 \mathrm{~V}$ and $\mathrm{K} 7 \mathrm{~V})$ spanning 4.5 years of ROSAT HRI data, suggestive of an activity cycle in X-rays. Some weak statistical evidence of solar-like cycles in lower-activity stars was previously reported by Hempelmann et al. (1996) using the RASS observations of the stars with known chromospheric cycles.

To study whether cyclical, high-amplitude variability of the coronal emission is also present on stars other than the Sun, three stellar systems have been monitored since the beginning of the XMM-Newton observatory. With a homogeneous temporal basis of almost 7 years, they represent the best available data set for the study of long-term variability and cycle in stars. The three systems monitored are HD 81809 (discussed in the present paper), $\alpha$ Cen (Robrade et al. 2005) and 61 Cyg (Hempelmann et al. 2006). 
Of the three, HD 81809 is the one with the longest available observational baseline and the one which shows the clearest evidence to date of a cyclical behavior.

The initial results of the observations of HD 81809 were presented by Favata et al. (2004, Paper I). High amplitude variability was evident in the data from 2001 to 2003 discussed in Paper I, with some coherence with the known chromospheric cycle of this star. However, no evidence of a coherent minimum was present in the data up to 2003, which made it impossible to distinguish a cyclic behavior from a simpler long-term trend in the star's activity level; the short data set was therefore completely inadequate for any study of the relation between the chromospheric and coronal activity variations. In this paper we present the results from all available observations, spanning 2001 to 2007, from which the cyclical behavior of the star's corona is evident with little if any remaining ambiguity.

\section{Characteristics of HD 81809}

While considered in the past as a candidate solar twin, HD 81809 is in fact a close visual binary with a separation at apoastron of about 0.4 arcsec and a period of about $35 \mathrm{yr}$ (Pourbaix 2000). The masses of the two components are $M_{1}=1.7 \pm 0.64 M_{\odot}$ and $M_{2}=1.0 \pm 0.25 M_{\odot}$, with spectral types G2 and G9 and apparent magnitudes $V_{1}=5.8$ and $V_{2}=6.8$ respectively. Both components are slow rotators, with $v \sin i=3 \mathrm{~km} \mathrm{~s}^{-1}$ (Soderblom 1982). More details regarding the characteristics of our star can be found in Paper I. HD 81809 has more recently been included in the high resolution spectroscopic survey of Takeda et al. (2005), who determined a photospheric Fe abundance of $[\mathrm{Fe} / \mathrm{H}]=-0.34$.

The binary nature of HD 81809 does not appear to be a problem for our study, as the very well defined chromospheric cycle reported by Baliunas et al. (1995) shows that the activity-related emission is most likely dominated by one of the two components. Also, the large physical separation of the two stars makes this a non-interacting system as also shown by the low rotational velocity mentioned above.

HD 81809 is one of the stars in the Mt. Wilson program showing a very clear cyclic behavior with high consistency from one cycle to the other. The chromospheric cycle of HD 81809 appears rather similar to the Sun's but with a shorter period of 8.2 yr. The available Mt. Wilson $S$ index observations are plotted in Fig. 1. The binary is not resolved, either in the Mt. Wilson data nor in the X-ray observations described below.

\section{Observations}

To sample the cyclic behavior of HD 81809, and considering the chromospheric cycle duration of 8.2 years, we have adopted a strategy based on two observations per year, spaced by 6 months. Given the visibility constraints of the XMM-Newton observatory, the observations are executed each year in the Spring and in the Fall (typically in April-May-June and September-OctoberNovember). The observations were executed regularly starting in April 2001, with the exception of the Spring 2006 season, in which a technical problem with the scheduling of XMM-Newton prevented the observation from being carried out. The length of the observation has been adapted along the program to track the large variation in luminosity shown by the target. Observations up to May 2003 were taken with the medium filter, while the later ones are taken with the thick filter due to a policy change of the observatory.

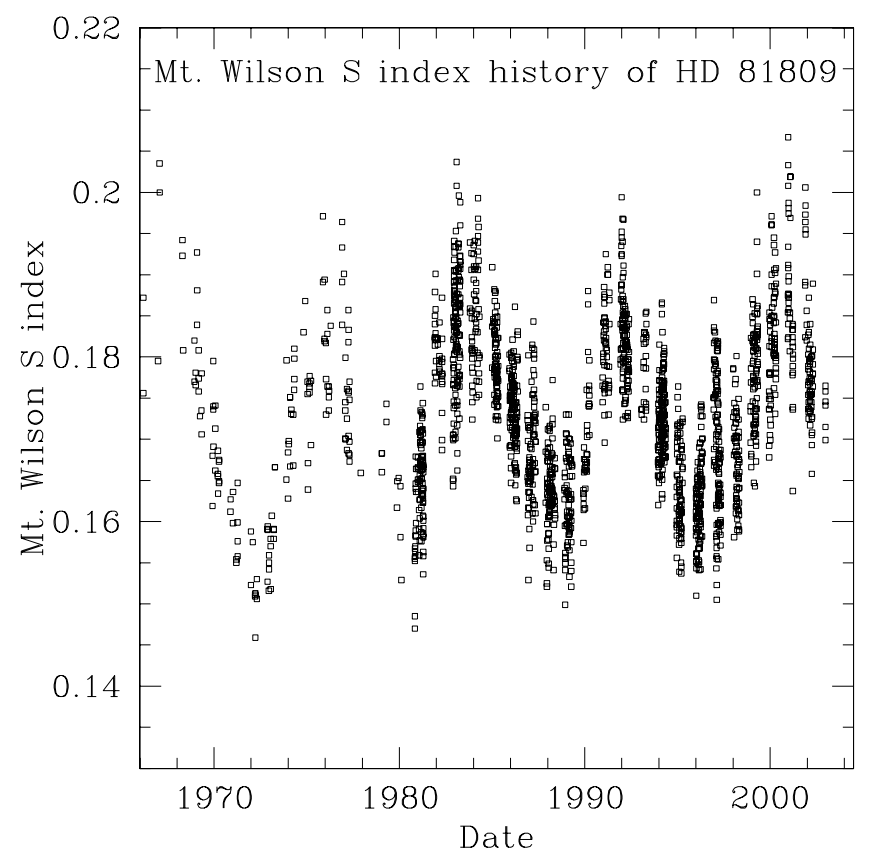

Fig. 1. Evolution of the chromospheric activity of HD 81809 in the Mt. Wilson $S$ index data, from 1966 to the end of 2002 (see Baliunas et al. 1995, for details, data courtesy of S. Baliunas).

In addition to the Mt. Wilson data, the Solar-Stellar Spectrograph (SSS) program at Lowell Observatory (Hall \& Lockwood 1995) has accumulated over a decade of Ca II H\&K observations of HD 81809. The SSS records the $H K$ spectrum from $[\lambda \lambda]$ 3860-4010 $\AA$ at a resolution of 12000 , and Hall et al. (2007) have described the calibration of the spectra to physical flux $\left(\log R_{H K}^{\prime}\right)$, and Mt. Wilson $S$. The raw CCD frames are reduced using an IDL package developed for the SSS data. Continuing the HD 81809 time series since the termination of the Mt. Wilson observations in 2003, the SSS observations revealed the expected minimum of the well-established 8.2-year cycle in 2004 , followed by a steady rise in activity in 2005-2007. In the following, for our comparison between the coronal and chromospheric variability of HD 81809 we use this data which has a full temporal overlap with our XMM-Newton monitoring program.

The X-ray data presented in this paper have been processed homogeneously using the SAS package. A consistent approach has been adopted in which high background time intervals are first removed from the observation, and then light curves and spectra are extracted. The pn and MOS spectra are simultaneously fit, using XSPEC, with a single temperature optically thin plasma model (APEC). The metal abundance has been frozen at $Z=0.3 Z_{\odot}$, to reduce the number of free parameters and allow a consistent comparison among the different data sets. The interstellar absorption from the fit is consistent with a zero value.

All observations (with the exception of the June 2002 data set) are well fit with a single-temperature model, with a modest range in best-fit temperature which is however well correlated with the star's X-ray luminosity. To allow a homogeneous analysis of all data sets, a one-temperature fit has been imposed also to the June 2002 data, even though the $\chi^{2}$ of the fit yields a very low probability.

For all observations X-ray luminosities were computed in two bands: a ROSAT-like $0.2-2.5 \mathrm{keV}$ band and for a Yohkohlike $0.73-3.5 \mathrm{keV}$ band. The resulting spectral parameters for the complete observation timeline are reported in Table 1, and 
Table 1. Best-fit spectral parameters for the 10 XMM-Newton observations of HD 81809 discussed here, spanning 4.5 years.

\begin{tabular}{ccccc}
\hline \hline Date & $\begin{array}{c}\text { pn rate } \\
\text { cts s}^{-1}\end{array}$ & $\begin{array}{c}L_{\mathrm{X}}(0.2-2.5 \mathrm{keV}) \\
\mathrm{erg} \mathrm{s}^{-1}\end{array}$ & $\begin{array}{c}L_{\mathrm{X}}(0.73-3.5 \mathrm{keV}) \\
\mathrm{erg} \mathrm{s}^{-1}\end{array}$ & $\begin{array}{c}k T \\
\mathrm{keV}\end{array}$ \\
\hline $2001-04-25$ & $0.1830 \pm 0.055$ & $3.85 \times 10^{28}$ & $1.46 \times 10^{28}$ & 0.34 \\
$2001-11-01$ & $0.3418 \pm 0.024$ & $6.42 \times 10^{28}$ & $2.91 \times 10^{28}$ & 0.40 \\
$2002-06-06$ & $0.7456 \pm 0.037$ & $1.78 \times 10^{29}$ & $8.88 \times 10^{29}$ & 0.81 \\
$2002-11-02$ & $0.2455 \pm 0.024$ & $5.31 \times 10^{28}$ & $2.31 \times 10^{28}$ & 0.39 \\
$2003-05-03$ & $0.1522 \pm 0.017$ & $3.37 \times 10^{28}$ & $1.36 \times 10^{28}$ & 0.36 \\
$2003-11-22$ & $0.0744 \pm 0.012$ & $2.05 \times 10^{28}$ & $7.33 \times 10^{27}$ & 0.33 \\
$2004-04-30$ & $0.0452 \pm 0.002$ & $1.32 \times 10^{28}$ & $4.59 \times 10^{27}$ & 0.31 \\
$2004-11-09$ & $0.0816 \pm 0.003$ & $1.67 \times 10^{28}$ & $6.57 \times 10^{27}$ & 0.33 \\
$2005-05-03$ & $0.0574 \pm 0.002$ & $1.41 \times 10^{28}$ & $4.65 \times 10^{27}$ & 0.32 \\
$2005-10-30$ & $0.0753 \pm 0.002$ & $1.81 \times 10^{28}$ & $7.56 \times 10^{27}$ & 0.32 \\
$2006-11-06$ & $0.1993 \pm 0.004$ & $4.28 \times 10^{28}$ & $1.71 \times 10^{28}$ & 0.36 \\
$2007-05-04$ & $0.2220 \pm 0.005$ & $5.48 \times 10^{28}$ & $2.24 \times 10^{28}$ & 0.38 \\
\hline
\end{tabular}

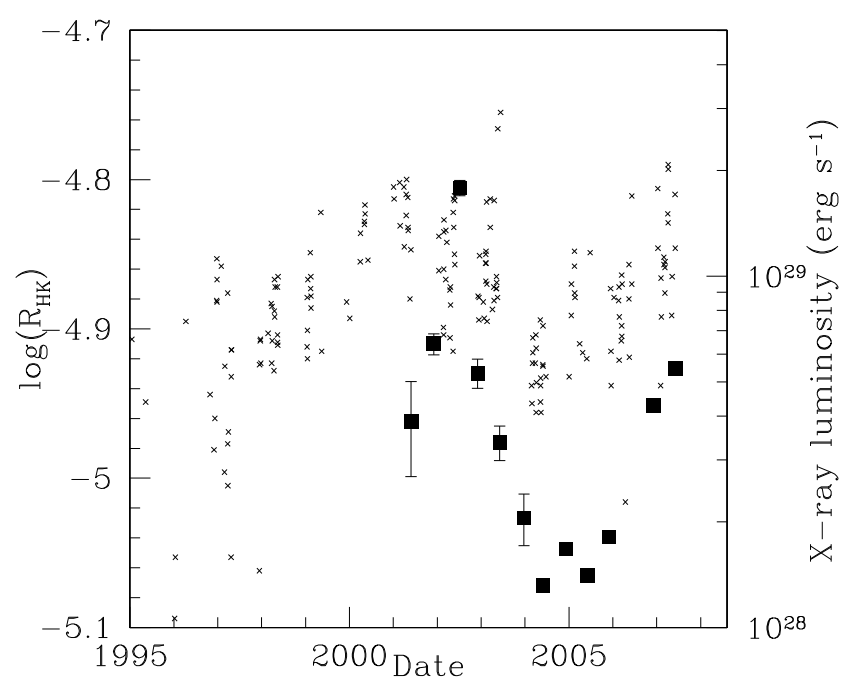

Fig. 2. Evolution of the mean (averaged over the duration of each XMM-Newton observation, each spanning a few hours) X-ray luminosity in the $0.2-2.5 \mathrm{keV}$ (ROSAT) band (squares, right hand scale) of HD 81809 from April 2001 to May 2007 together with the chromospheric Ca II activity measured by the SSS at Lowell (crosses, left hand scale). For the X-ray data points taken in 2004-2007 the uncertainty on the $\mathrm{X}$-ray luminosity is smaller than the symbol size.

a long-term light curve of the X-ray luminosity of HD 81809 is plotted (together with the $S$ index) in Fig. 2. None of the observations (with the exception of the June 2002 data point, discussed below) shows visible short-term variability, and each shows a flat light curve.

The observation of June 2002 is discussed in detail in Paper I. Briefly, although the light curve (which however only spans approximately two hours) shows no evidence of flare-like variability, the spectral characteristics, with a second temperature component at $T_{2}=1.3 \mathrm{keV}$ and comparable emission measure, suggest a period of enhanced, flare-like activity which drives the $\mathrm{X}$-ray luminosity above the average value for the season. Also, significant short-term variability is present in the June $2002 \mathrm{ob}-$ servation (as e.g. evident from Fig. 3 of Paper I), with amplitude of about $30 \%$ in the approximately $2 \mathrm{~h}$ spanned by the observation, indicative of a period of high activity.
As evident from Fig. 2, the first data point (of April 2001) is affected by a much larger uncertainty than the other points. The reason for this (again discussed in detail in Paper I) is a much higher background level across the complete observation, which, coupled with the short duration of the observation, resulted in a high noise level which could not be filtered out.

\section{Discussion}

The available long baseline of X-ray observations of HD 81809 for the first time allows us to state that HD 81809 shows longterm X-ray variability consistent with its regular chromospheric activity cycle. From a maximum that took place around 2002, the X-ray luminosity decreased to a broad minimum in 2004-2005, and is now rapidly increasing toward a new maximum. The latest observation, taken in May 2007, has an X-ray luminosity very close to the values observed in late 2001, and the maximum amplitude observed, if one ignores the apparently anomalous June 2002 observation, is about a factor of 6 . If one includes the June 2002 observation, the peak-to-peak variability amplitude is about a factor of 10 in the ROSAT band and a factor of about 100 in the Yohkoh-like band.

The coherent behavior of the observed variability clearly shows that one is not in the presence of high-amplitude stochastic variability but rather of a coherent variation in the coronal state of the star, with a time scale of several years, and with an amplitude not dissimilar to the cycle observed in the Sun.

The duration of the chromospheric cycle is well established at 8.2 years. Our X-ray data do not (yet) cover this time span, and therefore we have not yet sampled a complete cycle. The $\mathrm{X}$-ray data points in Fig. 2 may give the visual impression of a complete cycle, of a shorter duration, having been covered. However, it is likely that the maximum around 2001 and 2002 has not been properly sampled by our observations: the first point in April 2001 is affected by strong background, and the June 2002 point is likely affected by enhanced, flaring-like activity, resulting in an apparently very sharp maximum which is likely not real. In the Sun the maximum is normally rather broad and lasts a couple of years, and significant rotational modulation of the X-ray luminosity is present at solar maximum (with an amplitude up to $20 \%$ the total cycle amplitude), so that our single sample around the cycle maximum for HD 81809 may be affected by rotation-induced variability. On the other hand the 
minimum of the cycle is well sampled, and appears broad, with a duration of about 1.5-2 years. A further indication of the fact that the June 2002 point is unlikely to be the true X-ray cycle maximum is the apparent shift between the coronal and chromospheric maximum, as opposed to a good correlation between the chromospheric and coronal minima.

Based on both the observed chromospheric and coronal light curves, the maximum is expected in the next two years. To properly sample the period of maximum coronal activity, and to disentangle the short-term variability which is more likely to be present in periods of enhanced activity, we plan to ask for a more frequent monitoring of the star in upcoming XMM-Newton announcements of opportunity.

\subsection{A solar-like cycle?}

HD 81809 is not a strict solar twin, as it is somewhat more massive $\left(M=1.7 M_{\odot}\right)$ and more evolved, with a radius of $R \simeq 3 R_{\odot}$. It is also a member of a binary system, with smaller, less massive secondary component. However, the coherent, cyclic variability observed shows that the activity of the system is dominated by only one component in the system, which allows us to ignore the other component for the purpose of the present discussion. We assume in the following that the more massive, larger primary dominates the X-ray activity from HD 81809, an assumption which will be justified a posteriori as the solar-radius secondary is too small to justify the observed levels of activity with solar-like active regions and filling factors smaller than 1.

The X-ray luminosity of HD 81809 is higher than the Sun's by about 1.5 orders of magnitude. If one however considers the surface flux (assuming that the X-ray emission is dominated by the brighter component in the binary) the overlap with the Sun is large, with maximum flux values (again not considering the June 2002 observation) slightly above the solar ones and minima about mid-way between the solar maximum and minimum values, as shown in Fig. 3.

To study whether indeed the cycle observed on HD 81809 can be explained in terms of a solar-like behavior we have applied the methodology developed in the context of the study of the "Sun as a star" (Orlando et al. 2000; Peres et al. 2000). As shown by Orlando et al. (2001) from the analysis of a sample of data collected with the Soft X-ray Telescope (SXT, Tsuneta et al. 1991) on board the Yohkoh satellite, the evolution of the coronal X-ray luminosity and temperature along the solar cycle can be explained in terms of varying coverage of quiet background regions $(\mathrm{QR})$, active regions (AR) and cores of active regions $(\mathrm{CO})$, the latter being hotter and brighter than the other components of the non-flaring corona. We have assumed, in our simulation, a minimum radius for the active component (the primary) of HD 81809 of $2 R_{\odot}$, as this is the more "challenging" case (the smaller the star, the higher the filling factors necessary to reproduce the observed level of emission). Therefore the nominal filling factors reported in the following for the various types of coronal structures are to be intended as upper limits to the actual values. In a number of cases we also report the relevant values for the nominal radius of the $\operatorname{star} R=3 R_{\odot}$.

The observed temperature and luminosity distribution of the solar corona at minimum can be explained with a surface coverage of AR of about $0.3 \%$, with no $\mathrm{CO}$ present. At solar maximum, the observed emission can be typically explained by a surface coverage by AR of about $50 \%$ and a further approximately $1 \%$ of $\mathrm{CO}$. We have explored whether the variability observed for HD 81809 along the cycle can be explained by a mixture of the same ingredients as in the Sun but in different proportions.

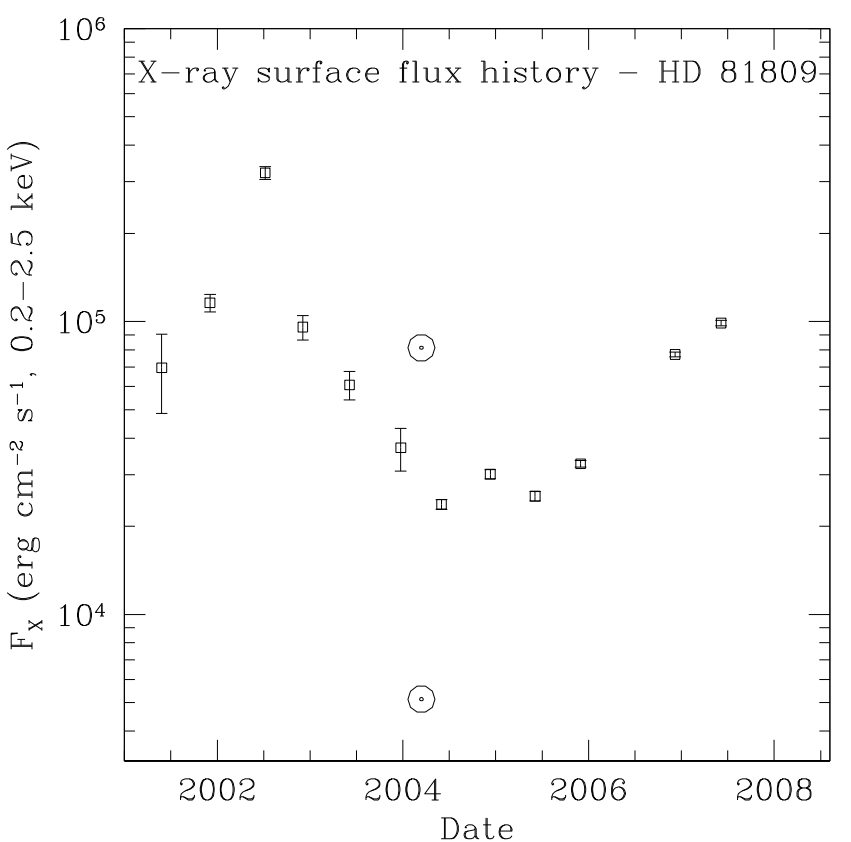

Fig. 3. Evolution of the X-ray surface flux (in the $0.2-2.5 \mathrm{keV}$ band) of HD 81809 from April 2001 to May 2007. The typical X-ray surface flux of the Sun at minimum and maximum of the cycle, in the ROSAT band, is also plotted.

To this end, we have considered the contribution of AR and $\mathrm{CO}$ to the luminosity and the spectrum of the Sun in the X-ray band as derived by Orlando et al. (2004), studying the long term evolution of a solar active region observed with Yohkoh/SXT from its emergence (July 5, 1996) to the decay phase (end of October 1996). Since the contribution of CO to the X-ray spectrum changes significantly during the active region evolution ${ }^{1}$, we have considered the $\mathrm{CO}$ during its early evolutionary phase, few days after the emergence of the active region.

Then, by simply assuming a permanent coverage of AR with a surface filling factor of about $60 \%\left(27 \%\right.$ for $\left.R=3 R_{\odot}\right)$ and a varying coverage of $\mathrm{CO}$, ranging from about $4 \%$ at the cycle minimum to about $40 \%$ at the cycle maximum (18\% for $R=$ $3 R_{\odot}$ ), we have synthesized the corresponding XMM-Newton spectra and reproduced rather accurately the observed range of variations of HD 81809 in terms of both the X-ray temperature and the luminosity. This is shown in Fig. 4, which shows the observed range of variation in coronal luminosity and temperature for both the Sun (data adapted from Orlando et al. 2001) and HD 81809 along their cycle, together with the synthetic values for HD 81809 obtained with the above approach. Table 2 reports the resulting spectral parameters for the synthetic spectra of HD 81809.

Figure 4 shows that while HD 81809 is more active, its cyclic behavior lies, in the temperature-luminosity plane, along an extension of the locus occupied by the Sun, and that therefore the phenomenology observed is compatible with being a simple extension of the solar case to a higher activity level. In the case of the Sun, the X-ray variability observed along the cycle is mainly due to the coverage of active regions, whereas the contribution from active region cores is mostly negligible. Our

1 In particular, Orlando et al. (2004) have found that the core of active region AR 7978 is characterized by an average temperature which varies from $\approx 6 \times 10^{6} \mathrm{~K}$ during the emergence of the active region (July $5-13,1996)$ to $\approx 2 \times 10^{6} \mathrm{~K}$ at the end of the core evolution circa one month later. 
Table 2. Best-fit spectral parameters for the synthetic spectra of HD 81809 derived assuming a different coverage of AR, CO, and flaring regions.

\begin{tabular}{|c|c|c|c|c|c|c|c|c|c|c|}
\hline \multirow[t]{2}{*}{ Phase } & \multicolumn{3}{|c|}{ Surface filling factor } & \multirow{2}{*}{$\begin{array}{c}k T_{1} \\
{[\mathrm{keV}]}\end{array}$} & \multirow{2}{*}{$\begin{array}{c}k T_{2} \\
{[\mathrm{keV}]}\end{array}$} & \multirow{2}{*}{$\begin{array}{c}E M_{1} \\
{\left[10^{50} \mathrm{~cm}^{-3}\right]}\end{array}$} & \multirow{2}{*}{$\begin{array}{c}E M_{2} \\
{\left[10^{50} \mathrm{~cm}^{-3}\right]}\end{array}$} & \multirow[t]{2}{*}{${\overline{\chi_{\mathrm{r}}}}^{2}$} & \multirow{2}{*}{$\begin{array}{c}L_{\mathrm{X}}(0.2-2.5 \mathrm{keV}) \\
{\left[\mathrm{erg} \mathrm{s}^{-1}\right]}\end{array}$} & \multirow{2}{*}{$\begin{array}{c}k T \\
{[\mathrm{keV}]}\end{array}$} \\
\hline & AR & $\mathrm{CO}$ & FL & & & & & & & \\
\hline Max & $60 \%$ & $40 \%$ & - & $0.24 \pm 0.03$ & $0.51 \pm 0.02$ & $2.0 \pm 0.4$ & $3.0 \pm 0.4$ & 0.65 & $5.35 \times 10^{28}$ & 0.40 \\
\hline Min & $60 \%$ & $4 \%$ & - & $0.16 \pm 0.01$ & $0.42 \pm 0.03$ & $0.51 \pm 0.07$ & $0.53 \pm 0.08$ & 0.70 & $9.70 \times 10^{27}$ & 0.29 \\
\hline Max + Flare & $60 \%$ & $40 \%$ & 0.0007 & $0.33 \pm 0.01$ & $1.26 \pm 0.02$ & $5.8 \pm 0.1$ & $8.1 \pm 0.2$ & 0.95 & $1.1 \times 10^{29}$ & 0.88 \\
\hline
\end{tabular}

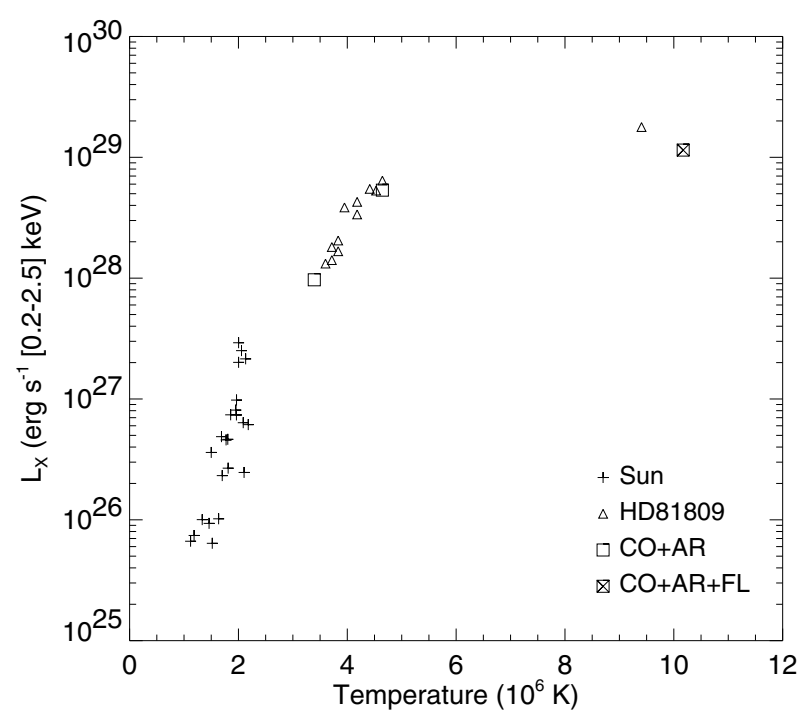

Fig. 4. The evolution of the coronal X-ray temperature and luminosity along the cycle in both the Sun (crosses, data adapted from Orlando et al. 2001) and HD 81809 (triangles). The figure also shows the synthetic values for HD 81809 obtained assuming a different coverage of $\mathrm{AR}$ and $\mathrm{CO}$ (squares); the crossed square marks the synthetic values for HD 81809 considering the contribution of a very intense flare (GOES class X9) to the non-flaring corona at the maximum of the cycle.

modeling shows that, in the case of HD 81809 the variability along the cycle is caused by the active region cores, which at the maximum of activity for HD 81809 cover almost half of the star. Figure 5 shows the modeled distributions of emission measure vs. temperature, $\operatorname{EM}(T)$ at the maximum and minimum of the cycle. Note that the contribution from AR to the $\operatorname{EM}(T)$ distribution (and to the synthetic X-ray spectrum) is important close to the minimum of the cycle when it is comparable to the contribution from CO. On the other hand, Fig. 5 shows that the $\operatorname{EM}(T)$ distribution is largely dominated by $\mathrm{CO}$ at the maximum of the cycle, whereas the contribution from AR is negligible and the amount of their surface filling factor cannot be univocally determined.

Also in the temperature-luminosity diagram the June 2002 point is an outlier, being well separated from the cloud of values which represent the variability of HD 81809 along the cycle, and therefore likely to be a separate, flare-like phenomenon. This hypothesis is supported by the fact that adding the contribution of a very intense solar flare (a GOES class X9 flare observed with Yohkoh/SXT on November 2, 1992) close to the flare maximum to the synthetic X-ray spectra of HD 81809 at the maximum of its cycle, the synthetic values of X-ray luminosity and average coronal temperature reproduced are close to those observed (see crossed square in Fig. 4).

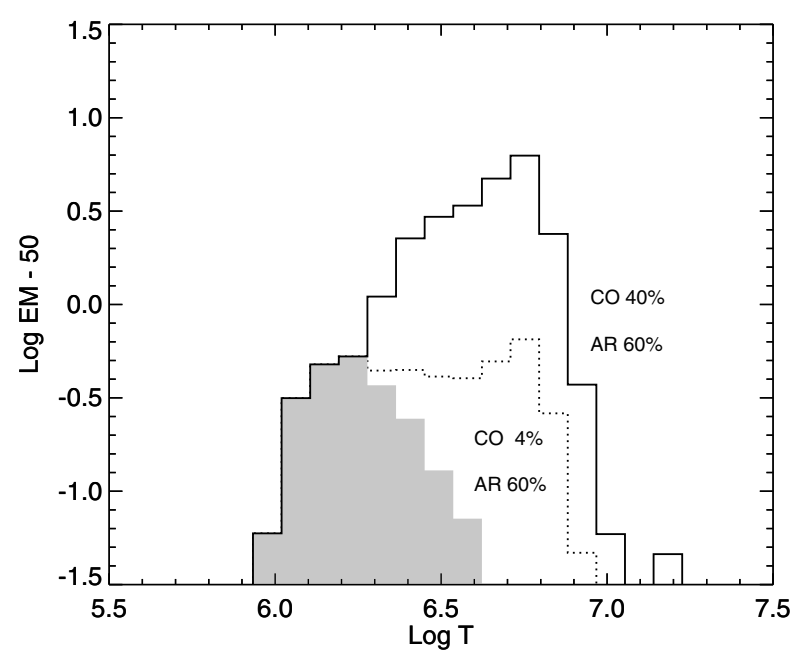

Fig. 5. The modeled emission measure vs. temperature distributions for HD 81809 at cycle maximum (solid histogram) and minimum (dotted histogram). The shaded area marks the contribution from AR.

The filling factors for the AR and the $\mathrm{CO}$ required to explain the observed variability along the HD 81809 cycle are large, but not unrealistic: the AR coverage is the same as the one observed in the Sun at the cycle maximum, while the CO coverage at maximum, while much higher than the one observed in the Sun, is still plausible.

\section{Conclusions}

We have presented the results from the first 7 years of monitoring of the solar-like star HD 81809 (a solar-type star with a well defined cycle in its chromospheric activity) with XMM-Newton, showing strong evidence of cyclic variability with an amplitude of at least 6 in the ROSAT $0.2-2.5 \mathrm{keV}$ band (and even more considering the outlier observed in June 2002). Our long-term XMM-Newton program has therefore for the first time resulted in the clear detection of a coronal cycle in a star other than the Sun.

Our observations have however not yet covered a full period of the observed chromospheric cycle. The coverage at the cycle maximum is scarce, and it may be affected by variability of the X-ray emission induced by rotational modulation, so that the single data point does not yet allow us to understand what is the duration of the maximum phase, and to which level is the stochastic variability enhanced with respect to the other phases. The minimum is on the other hand well covered, and it shows a very low level of stochastic variability both in terms of luminosity (a factor <2) and temperature, similar to what is observed in the Sun at the minimum.

At the maximum of activity, our simulations show that the $E M(T)$ distribution is dominated by $\mathrm{CO}$ (see Fig. 5) which 
determine the values of average temperature and emission measure reported in Fig. 4, whereas the contribution from AR to the total coronal emission is negligible. At the maximum, therefore, only the contribution from $\mathrm{CO}$ can be univocally determined, whereas for the AR the quoted value of $60 \%$ is in fact an upper limit to their contribution. When the star is at the minimum of the cycle, the contributions of $\mathrm{CO}$ and $\mathrm{AR}$ to the $E M(T)$ are comparable; changing their relative weight leads to a rapid change in the average temperature. In this case, therefore, the contributions of $\mathrm{CO}$ and AR are reasonably constrained: a doubling of the contribution of $\mathrm{CO}$ from $4 \%$ to $8 \%$ would lead to an increase in the X-ray luminosity of about $40 \%$.

Therefore, even if the absolute activity level of HD 81809 at its minimum is much higher than the Sun at minimum, its behavior is very similar. This is somewhat remarkable in that our model shows that a significant fraction of HD 81809 at minimum is covered by active regions, unlike the Sun which at minimum is dominated by coronal holes. In the future we plan to study in detail the upcoming maximum, with more frequent observations, to decouple the effect of stochastic variability and of rotational modulation of the X-ray emission.

HD 81809 is the subject of a long-term monitoring program performed with the XMM-Newton observatory (which also includes $\alpha$ Cen and $61 \mathrm{Cyg}$ ). Two more years of observations (at six months cadence) are already planned on the same target, and we plan to re-propose the target in future XMM-Newton AOs, to ensure the continuous monitoring. The continuation of the present program will allow us to shed new light on a very basic phenomenon of the Sun, its activity cycles, by studying how similar, somewhat more active stars behave.
Acknowledgements. G.M., S.S. and S.O. acknowledge the partial support of the ASI-INAF contracts I/023/05/0, I/015/07/0 and of MUR. J.H. acknowledges support from the National Science Foundation under grant ATM-0447159. This paper is based on observations obtained with XMM-Newton, an ESA science mission with instruments and contributions directly funded by ESA Member States and the USA (NASA). We would like to thank R. A. Donahue as well as the colleagues who have supported the first XMM-Newton proposal, namely S. Baliunas, A. Collier Cameron, M. Güdel, F. R. Harnden, R. Rosner, R. Stern, K. Strassmeier and F. Walter.

\section{References}

Acton, L. 1996, in Cool Stars, Stellar Systems, and the Sun, ed. R. Pallavicini, \& A. K. Dupree, ASP Conf. Ser., 109, 45

Baliunas, S. L., Donahue, R. A., Soon, W. H., et al. 1995, ApJ, 438, 269

Favata, F., Micela, G., Baliunas, S., et al. 2004, A\&A, 418, L13

Hempelmann, A., Schmitt, J. H. M. M., \& Stȩpieǹ, K. 1996, A\&A, 305, 284

Hempelmann, A., Schmitt, J. H. M. M., Baliunas, S. L., \& Donahue, R. A. 2003, A\&A, 406, L39

Hempelmann, A., Robrade, J., Schmitt, J. H. M. M., et al. 2006, A\&A, 460, 261 Judge, P. G., \& Saar, S. H. 2007, ApJ, 663, 643

Orlando, S., Peres, G., \& Reale, F. 2000, ApJ, 528, 524

Orlando, S., Peres, G., \& Reale, F. 2001, ApJ, 560, 499

Orlando, S., Peres, G., \& Reale, F. 2004, A\&A, 424, 677

Peres, G., Orlando, S., Reale, F., Rosner, R., \& Hudson, H. 2000, ApJ, 528, 537 Pourbaix, D. 2000, A\&AS, 145, 215

Robrade, J., Schmitt, J. H. M. M., \& Favata, F. 2005, A\&A, 442, 315

Schmitt, J. H. M. M., \& Liefke, C. 2004, A\&A, 417, 651

Soderblom, D. R. 1982, ApJ, 263, 239

Stern, R. 1998, in Cool Stars, Stellar Systems and the Sun, ed. R. A. Donahue, \& J. A. Bookbinder (San Francisco: ASP), ASP Conf. Ser., 154, 223

Takeda, Y., Ohkubo, M., Sato, B., Kambe, E., \& Sadakane, K. 2005, Publ. Astr. Soc. Japan, 57, 27

Tsuneta, S., Acton, L., Bruner, M., et al. 1991, Sol. Phys., 136, 37 